\title{
Is Random Close Packing of Spheres Well Defined?
}

\author{
S. Torquato $(1)(a)$, T. M. Truskett ${ }^{(2)}$, and P. G. Debenedetti ${ }^{(2)}$ \\ (1) Department of Chemistry and Princeton Materials Institute, Princeton University, Princeton, NJ 08544 \\ (2) Department of Chemical Engineering, Princeton University, Princeton, NJ 08544
}

\begin{abstract}
Despite its long history, there are many fundamental issues concerning random packings of spheres that remain elusive, including a precise definition of random close packing ( $\mathrm{RCP})$. We argue that the current picture of RCP cannot be made mathematically precise and support this conclusion via a molecular dynamics study of hard spheres using the Lubachevsky-Stillinger compression algorithm. We suggest that this impasse can be broken by introducing the new concept of a maximally random jammed state, which can be made precise.
\end{abstract}

5.20.-y, 61.20.-p

Random packings of identical spheres have been studied by biologists, materials scientists, engineers, chemists and physicists to understand the structure of living cells, liquids, granular media, glasses and amorphous solids, to mention but a few examples. The prevailing notion of random close packing (RCP) is that it is the maximum density that a large, random collection of spheres can attain and that this density is a universal quantity. An anonymous author summarizes this traditional view as follows: "ball bearings and similar objects have been shaken, settled in oil, stuck with paint, kneaded inside rubber balloons - and all with no better result than (a packing fraction of) ... 0.636." [1]

One aim of this paper is to reassess this commonly held view. First, we observe that there exists ample evidence in the literature (in the form of actual and computer experiments) to suggest strongly that the RCP state is ill-defined and, unfortunately, dependent on the protocol employed to produce the random packing as well as other system characteristics. In a classic experiment, Scott and Kilgour [2] obtained the RCP value $\phi_{c} \approx 0.637$ by pouring ball bearings into a large container, vertically vibrating the system for sufficiently long times to achieve maximum densification, and extrapolating the measured volume fractions to eliminate finite-size effects. Important dynamical parameters for this experiment include the pouring rate and both the amplitude and frequency of vibration. The key interactions are interparticle forces, including (ideally) repulsive hard-sphere interactions, friction between the particles (which inhibits densification), and gravity. It is clear that the final volume fraction can depend sensitively on these system characteristics. Indeed, in a recent experimental study [3], it was shown that one can achieve denser (partially crystalline) packings when the particles are poured at low rates into horizontally shaken containers.

Computer algorithms can be used to generate and study idealized random packings, but the final states are clearly protocol-dependent. For example, a popular rate-dependent densification algorithm [4 achieves $\phi_{c}$ between 0.642 and 0.649, a Monte Carlo scheme [5] gives $\phi_{c} \approx 0.68$, and a "drop and roll" algorithm [6] yields $\phi_{c} \approx 0.60$. It is noteworthy that in contrast to the last algorithm, the first two algorithms produce configurations in which either the majority or all of the particles are not in contact with one another. We are not aware of any algorithms that truly account for friction between the spheres.

However, we suggest that the aforementioned inconsistencies and deficiencies of RCP arise because it is an ill-defined state, explaining why, to this day, there is no theoretical determination of the RCP density. This is to be contrasted with the rigor that has been used very recently to prove that the densest possible packing fraction $\phi$ for identical spheres is $\pi / \sqrt{18} \approx 0.7405$, corresponding to the close-packed face-centered cubic (FCC) lattice or its stacking variants [7].

The term "close packed" implies that the spheres are in contact with one another with the highest possible coordination number on average. This is consistent with the view that RCP is the highest possible density that a random packing of close packed spheres can possess. However, the terms "random" and "close packed" are at odds with one another. Increasing the degree of coordination, and thus, the bulk system density, comes at the expense of disorder. The precise proportion of each of these competing effects is arbitrary and therein lies the problem. In what follows, we supply quantitative evidence of the ill-defined nature of RCP via computer simulations, and we propose a new notion, that of a maximally random jammed state.

A precise mathematical definition of the RCP state should apply to any statistically homogeneous and isotropic system of identical spheres (with specified interactions) in any space dimension $d$. Although we discard the term "close packed", we must retain the idea that the particles are in contact with one another, while maintaining the greatest generality. We say that a particle (or a set of contacting particles) is jammed if it cannot be translated while fixing the positions of all of the other particles in the system. The system itself is jammed if each particle (and each set of contacting par- 
ticles) is jammed [8]. This definition eliminates systems with "rattlers" (freely roaming caged particles) in the infinite-volume limit. We recognize that jammed structures created via computer algorithms [9] or actual experiments will contain a very small concentration of such rattler particles, the precise concentration of which is protocol-dependent. Thus, in practice, one may wish to accommodate this this type of a jammed structure, although the ideal limit described above is the precise mathematical definition of a jammed state that we have in mind. Nevertheless, it should be emphasized that it is the overwhelming majority of spheres that compose the underlying "jammed" network that confers rigidity to the particle packing.

Our definition of the maximally random jammed (MRJ) state is based on the minimization of an order parameter described below. The most challenging problem is quantifying randomness or its antithesis: order. A many-particle system is completely characterized statistically by the $N$-body probability density function $P\left(\mathbf{r}^{N}\right)$ associated with finding the system with configuration $\mathbf{r}^{N}$. Such complete information is never available and, in practice, one must settle for reduced information. From this reduced information, one can extract a set of scalar order parameters $\psi_{1}, \psi_{2}, \ldots, \psi_{n}$, such that $0 \leq \psi_{i} \leq 1, \forall i$, where 0 corresponds to the absence of order (maximum disorder) and 1 corresponds to maximum order (absence of disorder). The set of order parameters that one selects is unavoidably subjective, given that there is no single and complete scalar measure of order in the system.

However, within these necessary limitations, there is a systematic way to choose the best order parameters to be used in the objective function (the quantity to be minimized). The most general objective function consists of weighted combinations of order parameters. The set of all jammed states will define a certain region in the $n$ dimensional space of order parameters. In this region of jammed structures, the order parameters can divided up into two categories: those that share a common minimum and those that do not. The strategy is clear: retain those order parameters that share a common minimum and discard those that do not since they are conflicting measures of order. Moreover, since all of the parameters sharing a common minimum are essentially equivalent measures of order (there exists a jammed state in which all order parameters are minimized), choose from among these the one that is the most sensitive measure, which we will simply denote by $\psi$. From a practical point of view, two order parameters that are positively correlated will share a common minimum.

Consider all possible configurations of a $d$-dimensional system of identical spheres, with specified interactions, at a sphere volume fraction $\phi$ in the infinite-volume limit. For every $\phi$, there will be a minimum and maximum value of the order parameter $\psi$. By varying $\phi$ between zero and its maximum value (triangular lattice for $d=2$ and
FCC lattice for $d=3$ ), the locus of such extrema define upper and lower bounds within which all structures of identical spheres must lie. Figure 1 shows a schematic (not quantitative) plot of the order parameter versus volume fraction. Note that at $\phi=0$, the most disordered $(\psi=0)$ configurations of sphere centers can be realized. As the packing fraction is increased, the hard-core interaction prevents access to the most random configurations of sphere centers (gray region). Thus the lower boundary of $\psi$, representing the most disordered configurations, increases monotonically with $\phi$. The upper boundary of $\psi$ corresponds to the most ordered structures at each volume fraction, e.g. perfect open lattice structures $(\psi=1)$. Of course, the details of the lower boundary will depend on the particular choice of $\psi$. Nevertheless, the salient features of this plot are as follows: (1) all sphere structures must lie within the bounds and (2) the jammed structures are a special subset of the allowable structures [10]. We define the MRJ state to be the one that minimizes $\psi$ among all statistically homogeneous and isotropic jammed structures.

To support the aforementioned arguments, we have carried out molecular dynamics simulations using systems of 500 identical hard spheres with periodic boundary conditions. Starting from an equilibrium liquid configuration at a volume fraction of $\phi=0.3$, we compressed the system to a jammed state by the well-known method of Lubachevsky and Stillinger [9] which allows the diameter of the particles to grow linearly in time with a dimensionless rate $\Gamma$. Fig. 2a shows that the volume fraction of the final jammed states is inversely proportional to the compression rate $\Gamma$. A linear extrapolation of the data to the infinite compression rate limit yields $\phi \approx 0.64$, which is close to the supposed RCP value reported by Scott and Kilgour.

To quantify the order (disorder) in our jammed structures, we have chosen to examine two important measures of order: bond-orientational order and translational order [11]. The first is obtainable in part from the parameter $Q_{6}$ and the second is obtainable in part from the radial distribution function $g(r)$ (e.g., from a scattering experiment). To each nearest-neighbor bond emanating from a sphere, one can associate the spherical harmonics $Y_{l m}(\theta, \varphi)$, using the bond angles as arguments. Then $Q_{6}$ is defined by 12

$$
Q_{6} \equiv\left(\frac{4 \pi}{13} \sum_{m=-6}^{6}\left|\overline{Y_{6 m}}\right|^{2}\right)^{1 / 2}
$$

where $\overline{Y_{6 m}}$ denotes an average over all bonds. For a completely disordered system in the infinite-volume limit, $Q_{6}$ equals zero, whereas $Q_{6}$ attains its maximum value for space-filling structures $\left(Q_{6}^{F C C} \approx 0.575\right)$ in the perfect FCC crystal. Thus, $Q_{6}$ provides a measure of FCC crystallite formation in the system. For convenience we nor- 
malize the orientational order parameter $Q=Q_{6} / Q_{6}^{F C C}$ by its value in the perfect FCC crystal.

Scalar measures of translational order have not been well studied. For our purposes, we introduce a translational order parameter $T$ which measures the degree of spatial ordering, relative to the perfect FCC lattice at the same volume fraction. Specifically,

$$
T=\left|\frac{\sum_{i=1}^{N_{C}}\left(n_{i}-n_{i}^{\text {ideal }}\right)}{\sum_{i=1}^{N_{C}}\left(n_{i}^{F C C}-n_{i}^{\text {ideal }}\right)}\right|,
$$

where $n_{i}$ (for the system of interest) indicates the average occupation number for the spherical shell of width $a \delta$ located at a distance from a reference sphere that equals the $i$ th nearest-neighbor separation for the open FCC lattice at that density, $a$ is the first nearest-neighbor distance for that FCC lattice, and $N_{C}$ is the total number of shells (here we choose $\delta=.196$ and $N_{C}=7$ ). Similarly, $n_{i}^{i d e a l}$ and $n_{i}^{F C C}$ are the corresponding shell occupation numbers for an ideal gas (spatially uncorrelated spheres) and the open FCC crystal lattice. Observe that $T=0$ for an ideal gas (perfect randomness) and $T=1$ for perfect FCC spatial ordering.

The relationship between translational and bondorientational ordering has heretofore not been characterized. We have measured both $T$ and $Q$ for the jammed structures generated by the Lubachevsky-Stillinger algorithm and have plotted the results in the $Q-T$ plane in Fig. 2b [13]. This order plot reveals several key points. First, we observe that $T$ and $Q$ are positively-correlated and therefore are essentially equivalent measures of order for the jammed structures. Therefore, in seeking to determine the MRJ state using $T$ and $Q$, one would search for jammed structures that minimize $Q$, the more sensitive of the two measures. Our preliminary results indicate that the MRJ packing fraction $\phi_{M R J} \approx 0.64$ for 500 spheres using the Lubachevsky-Stillinger protocol. It should be noted, however, that a systematic study of other protocols may indeed find jammed states with a lower degree of order as measured by $Q$. Moreover, we notice that the degree of order increases monotonically with the jammed packing fraction [11]. These results demonstrate that the notion of RCP as the highest possible density that a random sphere packing can attain is ill-defined since one can achieve packings with arbitrarily small increases in volume fraction at the expense of small increases in order.

For purposes of comparison, we have included in the order plot of Fig. 2b results for the equilibrium hardsphere system for densities along the liquid branch and densities along the crystal branch, ending at the maximum close-packed FCC state 14]. Interestingly, the equilibrium structures exhibit the same monotonicity properties as the jammed structures, i.e., $T$ increases with increasing $Q$ and the degree of order increases with the packing fraction. Note that neither $Q$ nor $T$ are equal to unity along the equilibrium crystal branch because of thermal motion.

To summarize, we have shown that the notion of RCP is not well-defined mathematically. To replace this idea, we have introduced a new concept: the maximally random jammed state, which can be defined precisely once an order parameter $\psi$ is chosen. This lays the mathematical groundwork for studying randomness in dense packings of spheres and initiates the search for the MRJ state in a quantitative way not possible before. Nevertheless, significant challenges remain. First, new and efficient protocols (both experimental and computational) that generate jammed states must be developed. Second, since the characterization of randomness is in its infancy, the systematic investigation of better order parameters is crucial.

We thank F. H. Stillinger, T. Spencer, J. H. Conway, and M. Utz for many valuable discussions. S. T. was supported by the Engineering Research Program of the Office of Basic Energy Sciences at the US Department of Energy (DE-FG02-92ER14275) and the Guggenheim Foundation. He also thanks the Institute for Advanced Study in Princeton for the hospitality extended to him during his stay there. P. G. D. was supported by the Chemical Sciences Division of the Office of Basic Energy Sciences at the US Department of Energy (DE-FG0287ER13714). T. M. T. was supported by NSF.

(a) Corresponding author. Electronic mail address: torquato@matter.princeton.edu. Permanent address: Princeton Materials Institute and Dept. of Chemistry, Princeton University, Princeton, NJ 08544.

[1] See remarks published in Nature, 239488 (1972).

[2] G. D. Scott and D. M. Kilgour, Brit. J. Appl. Phys. 2 863 (1969).

[3] O. Pouliquen, M. Nicolas and P. D. Weldman, Phys. Rev. Lett. 793640 (1997).

[4] W. S. Jodrey and E. M. Tory, Phys. Rev. A 322347 (1985).

[5] J. Tobochnik and P. M. Chapin, J. Chem. Phys. 885824 (1988).

[6] W. M. Visscher and M. Bolsterli, Nature 239504 (1972).

[7] T. J. Hales (to be published).

[8] A fascinating open question is: How many jammed configurations exist at each packing fraction, i.e. what is the density of states for jammed configurations?

[9] B. D. Lubachevsky and F. H. Stillinger, J. Stat. Phys. 60561 (1990).

[10] The jammed structures with the lowest density for $d>$ 1 have yet to be identified. Examples of low-density jammed structures for $d=2$ have been noted by B. D. Lubachevsky, F. H. Stillinger, and E. N. Pinson, J. Stat. Phys. 64, 504 (1991). For $d=3$, the close-packed sim- 
ple cubic lattice (contained by rigid boundaries) with a packing fraction of $\phi=\pi / 6 \approx 0.52$ is an obvious example, but this is most likely not the lowest-density jammed structure.

[11] Other reasonable choices for order parameters were tested, including an information-theoretic entropy, and resulted in the same qualitative behavior as the order parameters described here $(Q$ and $T)$. All of these results, as well as the utility of such order parameters for general many-particle systems (including glasses), will be reported in a longer paper.

[12] P. J. Steinhardt, D. R. Nelson, and M. Ronchetti, Phys. Rev. B 28784 (1983).

[13] Our preliminary tests indicate that jammed structures with packing fractions in the range $(0.68<\phi<0.74)$ can be produced when very low compression rates $\left(\Gamma<10^{-3}\right)$ are employed. However, these structures are consistently more disordered (as determined by $T$ and $Q$ ) than the equilibrium FCC crystal structures at the same packing fraction.

[14] We also found that close-packed crystals composed of random sequences of FCC and HCP layer placements cannot be considered to be "random packings" by our chosen criteria. Both $Q$ and $T$ are not only higher than the minimum indicated in Fig. $2 \mathrm{~b}$ for the jammed structures but $Q$ lies in the range $0.84<Q<1$, depending on the stacking arrangement. 


\section{Fig. 1 Torquato et al.}

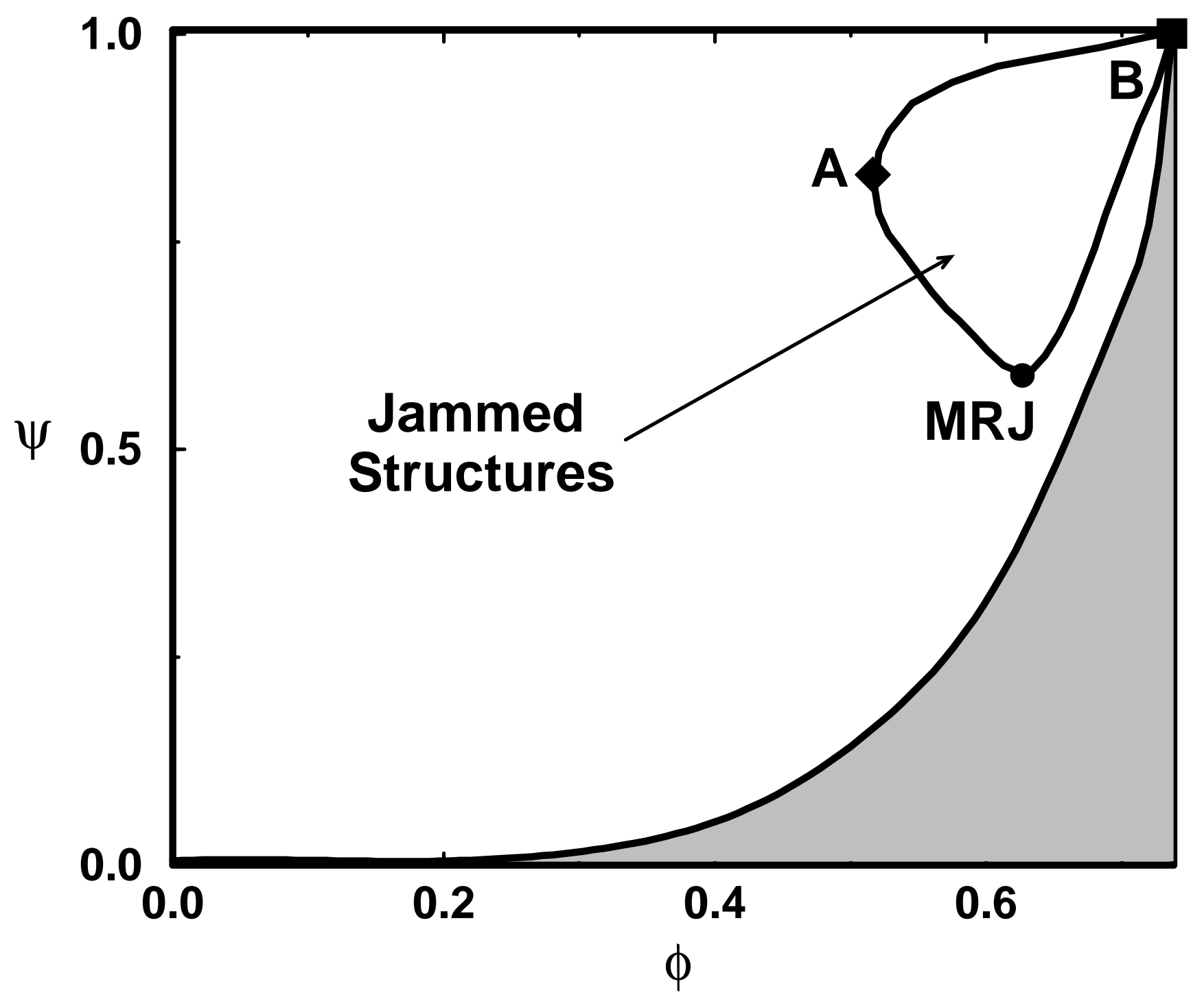

FIG. 1. A schematic plot of the order parameter $\psi$ versus volume fraction $\phi$ for a system of identical spheres with prescribed interactions. All structures at a given packing fraction $\phi$, must lie between the upper and lower bounds (white region); gray region is inaccessible. The boundary containing the subset of jammed structures is shown. The jammed structures are shown to be one connected set; although, in general, they may exist as multiply disconnected. Point A represents the jammed structure with the lowest density and point B represents the densest ordered jammed structure (e.g., close-packed FCC or hexagonal lattice for $d=3$, depending on the choice for $\psi$ ). The state which minimizes the order parameter $\psi$ is the maximally random jammed (MRJ) state. 

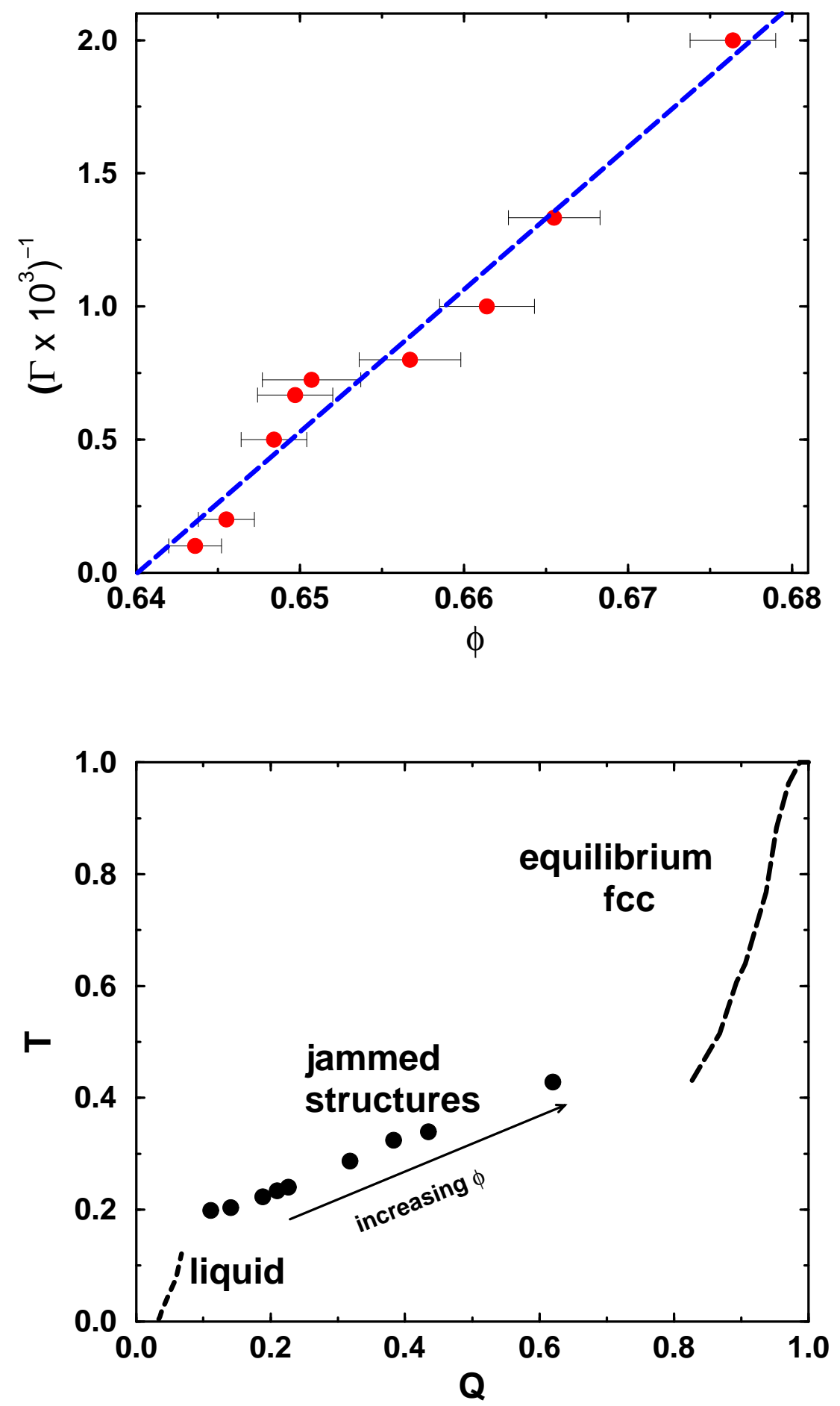

FIG. 2. Molecular dynamics simulation results for the hard-sphere system. (a) The reciprocal compression rate $\Gamma^{-1}$ versus the volume fraction $\phi$ of the final jammed state of hard spheres using the molecular dynamics compression protocol of Lubachevsky and Stillinger [9. The jammed state occurs when the diameters can no longer increase in time, the sphere collision rate diverges, and no further compression can be achieved after relaxing the configuration at the jammed volume fraction. Each point represents the average of 27 compressions, and the dashed line is a linear fit to the data, which yields $\phi \approx 0.64$ when $\Gamma^{-1}=0$. (b) The $Q-T$ plane for the hard-sphere system, where $T$ and $Q$ are translational and orientational order parameters, respectively. Shown are the average values for the jammed states of Fig. 2a (circles), as well as states along the equilibrium liquid (dotted) and crystal (dashed) branches. 